\title{
Bevacizumab in the treatment of ovarian cancer
}

\author{
This article was published in the following Dove Press journal: \\ Biologics:Targets and Therapy \\ II January 2011 \\ Number of times this article has been viewed
}

\section{Ramez N Eskander' \\ Leslie M Randall ${ }^{2}$ \\ 'Division of Gynecologic Oncology, Department of Obstetrics and Gynecology, University of California Irvine Medical Center, Orange, CA, USA; ${ }^{2}$ Division of Gynecologic Oncology, Department of Obstetrics and Gynecology, University of California, Irvine, Orange, CA, USA}

Correspondence: Leslie M Randall Assistant Professor, Division of Gynecologic Oncology, Department of Obstetrics and Gynecology, University of California, Bldg 56 Room 264, I0I The City Dr South, Orange, CA 92868, USA Tel + | 7|4-456-763 |

Fax + I 7|4-456-7754

Email Irandall@uci.edu
Abstract: Despite advances in surgical cytoreduction and cytotoxic chemotherapy, ovarian cancer continues to be the leading cause of death in women with gynecologic malignancy. Our understanding of the treatment of ovarian cancer was revolutionized with the discovery of platinum- and taxane-based adjuvant chemotherapy regimens. Since that time however, overall survival has been stable. Given the above, an emphasis has been placed on exploring alternative therapeutics. Recent research efforts have improved our understanding of the molecular biology of ovarian cancer and novel targeted treatment strategies have emerged. The most studied of these agents has been the monoclonal anti-vascular endothelial growth factor antibody bevacizumab. The purpose of this review is to discuss management issues related to the treatment of ovarian cancer, with a focus on the utilization of bevacizumab, summarizing applicable clinical trials, its potential benefits, and reported adverse events.

Keywords: bevacizumab, ovarian cancer, molecular targeted therapy, VEGF, angiogenesis

\section{Introduction}

Epithelial ovarian cancer (EOC) accounts for $25 \%$ of all malignancies affecting the female genital tract, and is the most lethal gynecologic malignancy. In the United States alone, a projected 21,880 new cases will be diagnosed, with 13,850 deaths. ${ }^{1}$ Advanced stage epithelial ovarian cancer is traditionally managed with surgery, followed by chemotherapy consisting of platinum and taxane. ${ }^{2}$ Patients with advanced disease who undergo successful cytoreductive surgery have a median survival of just over 4 years after completion of adjuvant chemotherapy. ${ }^{3}$ Unfortunately, the majority of these patients develop recurrent cancer, with selection of chemotherapy resistant clones. ${ }^{4}$

More recently, targeted therapies have been explored in the treatment of ovarian cancer and various potential therapeutic pathways, and biologic therapies have emerged. Specifically, anti-angiogenic agents have been the most studied and effective in the treatment of ovarian cancer. Angiogenesis is essential for tumor invasion and metastasis, and is required for tumor growth beyond 1-2 mm. This process requires the recruitment of vasculature and circulating endothelial cells, and pro-angiogenic mediators including vascular endothelial growth factor (VEGF). Ovarian cancer patients with elevated VEGF levels have been shown to have poorer overall prognosis, with VEGF levels being independent indicators of survival.

To date, bevacizumab, a recombinant human monoclonal antibody to the VEGF ligand, has been the most studied anti-angiogenic agent. The immediate mechanism of action of bevacizumab is to bind and inactivate VEGF, thereby inhibiting endothelial, and possibly tumor, cell activation and proliferation. ${ }^{6}$ In addition to denying tumors of 
nutrients, VEGF inhibition has been shown to induce vascular normalization, and to restore normal structure, function, and flow to the disorganized, leaky vessels characteristic of malignant tumors. These changes result in improved delivery of oxygen, nutrients, and cytotoxic chemotherapy to the tumor. ${ }^{7}$

Bevacizumab was first studied in patients with clear cell renal cell cancer, because of its unique VEGF-driven biology, and 4 other common solid tumors with high therapeutic need; colon, prostate, lung, and breast cancers. ${ }^{8}$ Phase III bevacizumab trials were then conducted in metastatic colorectal cancer (mCRC), ${ }^{9}$ metastatic nonsmall cell lung cancer (mNSCLC), ${ }^{10}$ and metastatic breast cancer (mBC), ${ }^{11}$ all of which met their primary endpoints, thus supporting Food and Drug Adminstgration (FDA) approval of bevacizumab for these indications. ${ }^{12}$ One of the next opportunities for approval will be in epithelial ovarian and primary peritoneal carcinomas.

\section{Current treatment regimens in ovarian cancer}

Currently, patients presenting with advanced stage ovarian cancer are managed with surgical resection followed by adjuvant platinum- and taxane-based chemotherapy. Our understanding of the therapeutic benefits of this regimen originated following the results of Gynecologic Oncology Group (GOG) protocol 111. ${ }^{13}$ This randomized trial illustrated an improvement in both progression free survival (PFS) and overall survival (OS) in patients treated with cisplatin and paclitaxel, in comparison to those treated using cisplatin and cyclophosphamide (PFS 18 vs 13 months, OS 38 vs 24 months, respectively). This study offered justification for a major change in the treatment of ovarian cancer.

Following completion of GOG 111, the GOG opened protocol $158 .{ }^{3}$ This was designed as a noninferiority trial comparing carboplatin and paclitaxel with cisplatin and paclitaxel. In previous nonrandomized trials the combination of carboplatin and paclitaxel was shown to be a less toxic and highly active combination regimen in the treatment of ovarian cancer. Ozols et al enrolled 792 eligible patients, with results showing decreased gastrointestinal, renal, and metabolic toxicities as well as decreased grade 4 leukopenia in the carboplatin-containing arm. ${ }^{3}$ Median PFS and OS did not differ significantly between study groups. The authors thus concluded that the combination of carboplatin and paclitaxel was less toxic, easier to administer, and not inferior to the previous standard of cisplatin and paclitaxel. Furthermore, in patients who were optimally cytoreduced (residual disease at completion of surgery $<1.0 \mathrm{~cm}$ ) the median survival was nearly 5 years in the carboplatin-containing arm.

In addition to intravenous (IV) therapy, the GOG also investigated intraperitoneal (IP) treatment options. GOG protocol 172 was designed to compare IV paclitaxel $\left(135 \mathrm{mg} / \mathrm{m}^{2}\right)$ over 24 hours with IV cisplatin $\left(75 \mathrm{mg} / \mathrm{m}^{2}\right)$ on day 2, vs IV paclitaxel $\left(135 \mathrm{mg} / \mathrm{m}^{2}\right)$ over 24 hours, followed by IP cisplatin $\left(100 \mathrm{mg} / \mathrm{m}^{2}\right)$ on day 2 and IP paclitaxel $\left(60 \mathrm{mg} / \mathrm{m}^{2}\right)$ on day 8 . A total of 6 courses would be administered every 3 weeks. ${ }^{14}$ All patients had optimally resected disease with residual tumor limited to less than or equal to $1 \mathrm{~cm}$ in size. The median survival for the IV and IP arms were 49.5 and 66.9 months respectively. The relative risk (RR) of death was 0.71 in the IP group $(P=0.0076)$. The authors however noted that tolerability for IP chemotherapy was a concern as grade 3 and 4 hematologic, metabolic, and gastrointestinal toxicities were significantly more common in the IP arm. In fact, only $42 \%$ of patients allocated to the IP arm completed 6 cycles of chemotherapy.

Unfortunately, despite the above advances in up-front adjuvant chemotherapy, disease recurrence continues to be problematic. Patients with recurrent disease are uncommonly cured, and thus comprise a population of patients for whom clinical trials and investigations into alternative therapies may be beneficial. Patients with disease recurrence 6 months after first-line therapy are termed "platinum sensitive", and have response rates in the range of $30 \%-40 \%$ to second-line agents. ${ }^{15}$ These patients typically receive a platinum-based regimen, often in combination with paclitaxel, gemcitabine, or pegylated liposomal doxorubicin. ${ }^{16}$ On the other hand, patients with disease recurrence within 6 months of up-front therapy, termed platinum resistant, and those with disease persistence/progression while on first-line platinum therapy, termed platinum refractory, are typically treated with nonplatinum-based regimens. ${ }^{15}$ Agents that can be considered in this setting include liposomal doxorubicin, topotecan, gemcitabine, and oral etoposide. ${ }^{17}$

\section{Bevacizumab}

As described above, despite multimodal chemotherapy after surgical cytoreduction, the minority of women with ovarian cancer are actually cured. Thus, exploration of alternative therapeutics has been a priority. Specifically, the study of angiogenesis in ovarian cancer, and the role of potential modulators of angiogenesis in its treatment have emerged as a promising strategy. ${ }^{18}$ Angiogenesis is the process of new 
blood vessel development and is essential for tumor growth beyond 100-200 $\mu \mathrm{m}$. This process is mediated through the VEFG family of growth factors and receptors. The immediate action of bevacizumab, the first FDA-approved monoclonal anti-angiogenic antibody, is to bind and inactivate VEGF, inhibiting endothelial cell proliferation and activation. The utility of VEGF in the treatment of ovarian carcinoma was initially explored in animal models, where VEGF blockade was shown to inhibit ascites formation and slow tumor growth. ${ }^{19}$ Numerous retrospective clinical studies have shown that VEGF polymorphisms, and increased intra-tumoral VEGF gene expression correlates with poor prognosis. ${ }^{20}$ Ovarian cancer tumor vasculature also tends to be structurally and functionally abnormal, leaky and tortuous, with immature endothelial cells. These cells have increased dependence on VEGF for survival in comparison to mature vasculature. $^{21,22}$

Two single-agent phase 2 bevacizumab trials have explored its utility in the treatment of recurrent, predominantly platinum-resistant, ovarian cancer. The first, GOG 170D, the largest single agent study, explored the use of bevacizumab at a dose of $15 \mathrm{mg} / \mathrm{kg}$ in patients with persistent or recurrent epithelial ovarian cancer or primary peritoneal cancer after 1-2 prior cytotoxic regimens. ${ }^{23}$ The study consisted of 62 eligible and assessable patients. Of these, $66.1 \%$ had received 2 prior cytotoxic chemotherapy regimens, and $41.9 \%$ were considered platinum resistant. Thirteen patients $(21.0 \%)$ experienced clinical responses ( 2 complete, 11 partial; median response duration, 10 months), and 25 (40.3\%) survived progression free for at least 6 months. Median PFS and OS were 4.7 and 17 months, respectively. The second was an industry-sponsored trial evaluating the efficacy and safety of bevacizumab in patients with platinum-resistant epithelial ovarian carcinoma or peritoneal serous carcinoma who had experienced disease progression during or within 3 months of discontinuing topotecan or liposomal doxorubicin. ${ }^{24}$ Enrolled patients received single-agent bevacizumab $15 \mathrm{mg} / \mathrm{kg}$ every 3 weeks, and nearly $84 \%$ were platinum resistant. Partial responses were observed in $15.9 \%$ with median PFS of 4.4 months (95\% CI: 3.1-5.5 months), and a median survival duration of 10.7 months at study termination. This was followed by 2 trials combining cytotoxic chemotherapy with bevacizumab in the treatment of recurrent disease. The trials showed response rates of $24 \%$ each and 6-month progressionfree rates of $50 \%$ and $56 \%$, respectively. ${ }^{25,26}$

The above phase 2 trials were then followed by 4 randomized phase 3 trials, looking at bevacizumab as a first-line agent and in disease recurrence. Three of these trials have completed accrual, namely GOG 218, International collaborative ovarian neoplasm (ICON) 7, and OCEANS. GOG 218 is a 3-arm placebo controlled study, with all patients receiving carboplatin and paclitaxel. In the first experimental arm, patients were treated with concurrent bevacizumab followed by placebo maintenance, while in the third experimental arm, patients received concurrent and maintenance bevacizumab (15 mg/kg every 3 weeks) for up to 16 doses. ${ }^{27} \mathrm{~A}$ total of 1873 patients were enrolled. Of these patients, 34\% were optimally cytoreduced, with $40 \%$ suboptimally debulked, and $26 \%$ being stage 4 . Preliminary data, as presented at the 2010 meeting for the American Society of Clinical Oncology (ASCO), showed a significant improvement in PFS in patients treated with concurrent and maintenance bevacizumab, 14.1 months vs 10.3 months in the placebo arm. Relative to arm 1 of the trial, the hazard ratio for first progression in the maintenance arm of the trial was 0.717 (95\% CI: $0.625-0.824, P<0.0001$ ). The OS data are not yet mature.

ICON-7 was a 2 -armed trial comparing carboplatin and paclitaxel (6 cycles) against carboplatin + paclitaxel + bevacizumab $(7.5 \mathrm{mg} / \mathrm{kg})$ every 3 weeks for 6 cycles, followed by 12 cycles of maintenance bevacizumab or disease progression, whichever occurred earlier. Data from this trial were presented in abstract form at the 2010 meeting of the European Society of Medical Oncology (ESMO). A total of 1528 women were randomized from 263 centers. Adverse events were reported as consistent with previous bevacizumab trials. More importantly, relative to arm 1 of the trial, the hazard ratio for disease progression in the bevacizumab arm was 0.81 (95\% CI: 0.70-0.94, $P<0.0041)$.

The remaining phase III trials target patients with recurrent disease. GOG 213, is a randomized trial comparing carboplatin and paclitaxel with or without bevacizumab in platinum sensitive relapsed ovarian cancer patients. It also includes a study of secondary cytoreductive surgery in eligible patients. OCEANS, an industry-conducted study, is a randomized, placebo- controlled trial, comparing carboplatin and gemcitabine with or without bevacizumab in the treatment of recurrent disease. ${ }^{7}$ This trial has completed accrual, but results are not available at this time (Table 1).

As with any new anti-tumor agent, the benefits of bevacizumab in the treatment of patients with ovarian cancer must be weighed against the side effects. Overall, bevacizumab is well tolerated, with a side effect profile unique to its mode of action. In a comprehensive review, Randall et al investigated 
Table I Randomized clinical trials evaluating bevacizumab in ovarian cancer

\begin{tabular}{|c|c|c|}
\hline Study & Trial design & Status \\
\hline \multicolumn{3}{|c|}{ First-line adjuvant therapy } \\
\hline GOG 218 & $\begin{array}{l}\text { Randomized, placebo-controlled, 3-arm, } \\
C T \pm \text { bev with } 18 \text {-cycle bev maintenance }\end{array}$ & $\begin{array}{l}\text { Completed accrual: HR } 0.717 \text { favoring the concurrent } \\
\text { and maintenance bevacizumab arm }\end{array}$ \\
\hline ICON-7 & $\begin{array}{l}\text { Randomized, } 2 \text {-arm, } C T \pm \text { bev with } \\
\text { 12-cycle bev maintenance }\end{array}$ & $\begin{array}{l}\text { Completed accrual: HR } 0.80 \text { favoring the concurrent } \\
\text { and maintenance bevacizumab arm }\end{array}$ \\
\hline \multicolumn{3}{|c|}{ Second-line therapy } \\
\hline GOG 213 & Randomized, CT \pm bev & Open to accrual \\
\hline OCEANS & Randomized, placebo-controlled, $\mathrm{CG} \pm$ bev & Completed accrual July 20 I0: Results not yet available \\
\hline
\end{tabular}

Abbreviations: CT, carboplatin/paclitaxel; CG, carboplatin/gemcitabine; GOG, Gynecologic Oncology Group; ICON, International Collaborative Ovarian Neoplasm; bev, bevacizumab.

the toxicities of bevacizumab in ovarian cancer patients, and their management. ${ }^{7}$ Table 2 lists the adverse effects that were included in the FDA-approved bevacizumab package insert. ${ }^{28}$

The most common toxicities include hypertension (grade 3-4 ranging from $0.3 \%$ to $14.8 \%$ of patients), proteinuria (usually grade 1-2), hemorrhage, arterial and venous thrombotic events, impaired wound healing, and gastrointestinal perforation. ${ }^{18,29}$ Treatment strategies for bevacizumab related hypertension have yet to be defined, but traditional antihypertensive agents including beta blockers, calcium channel blockers, and ACE inhibitors have successfully managed bevacizumab-related hypertension.

Bowel perforation is an additional feared adverse event and appears to be disease site-dependent, occurring in patients with $\mathrm{mCRC}$ and ovarian carcinoma. Ovarian cancer patients may be at increased risk due to the potential for bowel dysmotility and obstruction as a direct result of the disease process. Data from key phase II trials discussed previously indicated that patients who were heavily pretreated (3 or more previous cycles of cytotoxic chemotherapy) were at increased risk of bowel perforation. Although there were no reported gastrointestinal perforations (GIP) in GOG 170-D, eligible patients were limited to 1 or 2 previous chemotherapy regimens..$^{23}$ Conversely, the phase II study completed by Cannistra et al was terminated early due to the high rate of GIP in the patient population, $11.4 \%$ (5 of 44 patients). ${ }^{24}$

Table 2 Adverse events associated with bevacizumab

\begin{tabular}{ll}
\hline Adverse events & \\
\hline Common (mild) & Rare (serious) \\
\hline Headache & Nongastrointestinal fistula formation \\
Hypertension & $\begin{array}{l}\text { Arterial thromboembolic events (myocardial } \\
\text { infarction, cerebrovascular accident) }\end{array}$ \\
Proteinuria & $\begin{array}{l}\text { Gastrointestinal perforation } \\
\text { Wound healing complications } \\
\text { Reversible posterior leukoencephalopathy } \\
\end{array}$ \\
& syndrome \\
\hline
\end{tabular}

Importantly, all GIP occurred in patients receiving 3 or more previous chemotherapy regimens. We are awaiting final data from GOG 218 and ICON-7 to help elucidate risk in up-front treatment with bevacizumab. ${ }^{18}$

The management of bevacizumab toxicity requires individualized decision making that weighs drug efficacy against adverse event severity (grade) and potential longterm consequences of both the adverse event and the disease. The goals of therapy (palliative vs curative), potential alternative therapies, cost, and patient quality of life should all be factored into these decisions. Communicating to the patient the complexity of issues that inform treatment decisions can aid in the management of expectations as well.

\section{Conclusion}

In summary, our understanding of the complex molecular pathways that control tumor angiogenesis continues to improve. The anti-VEGF agent bevacizumab has shown activity in the treatment of ovarian cancer, with an overall acceptable toxicity profile and is currently listed as an active treatment for recurrent ovarian and primary peritoneal cancers by the National Comprehensive Cancer Network, but it is not yet FDA-approved for these indications. Bevacizumab has been shown to exhibit a $15 \%-20 \%$ response rate with up to $50 \%$ PFS at 6 months in several phase II trials..$^{23-26}$ Furthermore, the preliminary data from 2 phase III clinical trials, GOG 218 and ICON-7, show an improvement in PFS in the up-front treatment of ovarian cancer with the addition of bevacizumab. It is unclear, at this point in time, how these data will be applied clinically.

In the last decade, numerous biologic targets, including VEGF-inhibtors, epidermal growth factor receptor inhibitors, poly-ADP-ribose polymerase inhibitors, and anti-folate agents have been identified and studies are under way to determine their utility in the treatment of ovarian cancer. Future studies exploring potential biomarkers to aid in 
predicting response to therapy (analogous to HER2 in breast cancer) are of utmost importance, as this will allow us to better select patients for therapy.

\section{Disclosure}

The authors declare no conflicts of interest.

\section{References}

1. Jemal A, Siegel R, Xu J, Ward E. Cancer Statistics, 2010. CA Cancer J Clin. 2010;60:277-300.

2. Bookman M. Trials with impact on clinical management: first line. Int J Gynecol Cancer. 2009;19 Suppl 2:S55-S62.

3. Ozols R, Bundy B, Greer B, et al. Phase III trial of carboplatin and paclitaxel compared with cisplatin and paclitaxel in patients with optimally resected stage III ovarian cancer: a Gynecologic Oncology Group study. J Clin Oncol. 2003;21:3194-3200.

4. Monk B, Choi D, Pugmire G, et al. Activity of bevacizumab (rhuMAB VEGF) in advanced refractory epithelial ovarian cancer. Gynecol Oncol. 2005;96:902-905.

5. Folkman J. Tumor angiogenesis: therapeutic implications. N Engl J Med. 1971;285:1182-1186.

6. Burger R. Experience with bevacizumab in the management of epithelial ovarian cancer. J Clin Oncol. 2007;25:2902-2908.

7. Randall L, Monk B. Bevacizumab toxicities and their management in ovarian cancer. Gynecol Oncol. 2010;117:497-504.

8. Ferrara N, Hillan K, Gerber H, et al. Discovery and development of bevacizumab, an anti-VEGF antibody for treating cancer. Nat Rev Drug Discov. 2004;3:391-400.

9. Hurwitz H, Fehrenbacher L, Novotny W, et al. Bevacizumab plus irinotecan, fluorouracil, and leucovorin for metastatic colorectal cancer. N Engl J Med. 2004;350:2335-2342.

10. Sandler A, Gray R, Perry M, et al. Paclitaxel-carboplatin alone or with bevacizumab for non-small-cell lung cancer. $N$ Engl J Med. 2006;355: 2542-2550.

11. Miller K, Wang M, Gralow J, et al. Paclitaxel plus bevacizumab versus paclitaxel alone for metastatic breast cancer. N Engl J Med. 2007;357: 2666-2676.

12. Escudier B, Pluzanska A, Koralewski P, et al. Bevacizumab plus interferon alfa-2a for treatment of metastatic renal cell carcinoma: a randomised, double-blind phase III trial. Lancet. 2007;370:2103-2111.

13. McGuire W, Hoskins W, Brady M, et al. Cyclophosphamide and cisplatin compared with paclitaxel and cisplatin in patients with stage III and stage IV ovarian cancer. N Engl J Med. 1996;334:1-6.

14. Markman M, Bundy B, Alberts D, et al. Phase III trial of standarddose intravenous cisplatin plus paclitaxel versus moderately high-dose carboplatin followed by intravenous paclitaxel and intraperitoneal cisplatin in small-volume stage III ovarian carcinoma: an intergroup study of the Gynecologic Oncology Group, Southwestern Oncology Group, and Eastern Cooperative Oncology Group. J Clin Oncol. 2001; 19:1001-1007.
15. Cannistra S. Evaluating new regimens in recurrent ovarian cancer: how much evidence is good enough? J Clin Oncol. 2010;28:3101-3103.

16. Pujade-Lauraine E, Wagner U, Aavall-Lundqvist E, et al. Pegylated liposomal doxorubicin and carboplatin compared with paclitaxel and carboplatin for patients with platinum-sensitive ovarian cancer in late relapse. J Clin Oncol. 2010;28:3323-339.

17. Cannistra S. Is there a "best" choice of second-line agent in the treatment of recurrent, potentially platinum-sensitive ovarian cancer? J Clin Oncol. 2002;20:1158-1160.

18. Kumaran G, Jayson G, Clamp A. Antiangiogenic drugs in ovarian cancer. Br J Cancer. 2009;100:1-7.

19. Byrne A, Ross L, Holash J, et al. Vascular endothelial growth factor-trap decreases tumor burden, inhibits ascites, and causes dramatic vascular remodeling in an ovarian cancer model. Clin Cancer Res. 20039: 5721-5728.

20. Shen G, Ghazizadeh M, Kawanami O, et al. Prognostic significance of vascular endothelial growth factor expression in human ovarian carcinoma. Br J Cancer. 2000;83:196-203.

21. Kamba T, Tam B, Hashizume H, et al. VEGF-dependent plasticity of fenestrated capillaries in the normal adult microvasculature. Am J Physiol Heart Circ Physiol. 2006;290:H560-H576.

22. Fukumura D, Jain R: Tumor microvasculature and microenvironment: targets for anti-angiogenesis and normalization. Microvasc Res. 2007; 74:72-84.

23. Burger R, Sill M, Monk B, et al. Phase II trial of bevacizumab in persistent or recurrent epithelial ovarian cancer or primary peritoneal cancer: a Gynecologic Oncology Group Study. J Clin Oncol. 2007;25: 5165-5171.

24. Cannistra S, Matulonis U, Penson R, et al. Phase II study of bevacizumab in patients with platinum-resistant ovarian cancer or peritoneal serous cancer. J Clin Oncol. 2007;25:5180-5186.

25. Garcia A, Hirte H, Fleming G, et al. Phase II clinical trial of bevacizumab and low-dose metronomic oral cyclophosphamide in recurrent ovarian cancer: a trial of the California, Chicago, and Princess Margaret Hospital phase II consortia. J Clin Oncol. 2008;26:76-82.

26. Nimeiri H, Oza A, Morgan R, et al. Efficacy and safety of bevacizumab plus erlotinib for patients with recurrent ovarian, primary peritoneal, and fallopian tube cancer: a trial of the Chicago, PMH, and California Phase II Consortia. Gynecol Oncol. 2008;110:49-55.

27. Auranen A, Grénman S. Radiation therapy and biological compounds for consolidation therapy in advanced ovarian cancer. Int $J$ Gynecol Cancer. 2008;18 Suppl 1:44-46.

28. Zhu X, Wu S, Dahut W, et al. Risks of proteinuria and hypertension with bevacizumab, an antibody against vascular endothelial growth factor: systematic review and meta-analysis. Am J Kidney Dis. 2007;49: 186-193.

29. Izzedine H, Rixe O, Billemont B, et al. Angiogenesis inhibitor therapies: focus on kidney toxicity and hypertension. Am J Kidney Dis. 2007;50: 203-218.

\section{Publish your work in this journal}

Biologics: Targets \& Therapy is an international, peer-reviewed journal focusing on the patho-physiological rationale for and clinical application of Biologic agents in the management of autoimmune diseases, cancers or other pathologies where a molecular target can be identified. This journal is indexed on PubMed Central, CAS, EMBase, Scopus

\section{Dovepress}

and the Elsevier Bibliographic databases. The manuscript management system is completely online and includes a very quick and fair peerreview system, which is all easy to use. Visit http://www.dovepress. $\mathrm{com} /$ testimonials.php to read real quotes from published authors. 